\title{
Forum
}

\section{The Use of Radar Reflectors to Validate Radar Performance Calculations}

\author{
P. D. L. Williams and G. Bryant \\ (Open University, Milton Keynes)
}

DISCUSSION. There is renewed interest to compare actual radar performance at sea with that forecast by computational methods. This interest has partly been brought about by the recent availability of proprietary software in disc form using the methods outlined by L. V. Blake in (1969). ${ }^{1}$ There is a significant source of likely error over and above the usual argument and later agreement on the various losses as may be experienced on board ship or at a coastal radar site. The error is in taking the peak kcs (radar cross section), as may be stamped on a proprietary radar reflector (which may not even be qualified in terms of operating wavelength), and using this to enter the median RCs as called up by most forecasting programmes.

In addition to this is the further amplitude modulation of 'free space' radar performance as brought about by Lloyd's mirror. This may be allowed for by proper use of the propagation term ' $F$ ' (see Skolnik $1980^{2}$ and Kerr $1951^{3}$ ) but with a higher degree of uncertainty.

There is now a further complication in that the ' $F$ ' term was initially applied to targets at ranges where they were small compared to the likely angular dimensions of the lobes set up by sea reflection of the indirect wave as applied to aircraft. At sea, with ship targets, the extended nature of these in the vertical plane reduces the nulls such as may be found with a point target. This is illustrated in Fig. I by one of the trials results from Squires $(197 \mathrm{r})^{4}$ where one pair of curves ( $\mathrm{ra}$ and $\mathrm{Ib}$ ) corresponds to the unenhanced performance of a small target vessel. To the right is the performance obtained when the same vessel carried a corner reflector of peak echoing area estimated at $100 \mathrm{~m}^{2}$ at ' $\mathrm{X}$ ' Band ( $\mathrm{I} \mathrm{c}$ and $\mathrm{I}$ ). The results from this run $\mathrm{I}$ show the extended range obtained by use of a large reflector, but note how the modulation changes using the reflector in curves $(c)$ and $(d)$ as a function of boat heading.

In another run, shown in Fig. 2 using a reflector, the destructive interference is clearly visible at $1 \cdot 2-\mathrm{I} \cdot 3 \mathrm{n} . \mathrm{m}$. for a receding target but less for its approach run. Is this just a result of reflector orientation?

CONCLUSIONS. Radar calibration trials are best carried out by the use of conducting spheres or true omnidirectional targets but, if corner reflectors are to be used, due allowance must be made for their likely peak to mean ratio. Ideally, calibration trials should be arranged with only a sphere target; a host vessel badly corrupts results.

This effect is in addition to the further effect due to reduced height on the water compared to the ship it is intended to simulate. The peak-to-median ratio should be at least $7 \mathrm{~dB}$, preferably $10 \mathrm{~dB}$ (Williams, 1978) ( $^{5}$ and the effect of reduced height above sea level may be a further $10-20 \mathrm{~dB}$ as recommended in the correction curve given in the appropriate British Standard (HMSO, 1977) ${ }^{6}$ though this is more concerned with small ship visibility for safety reaso.ss than radar trial validation of forecast performance. 


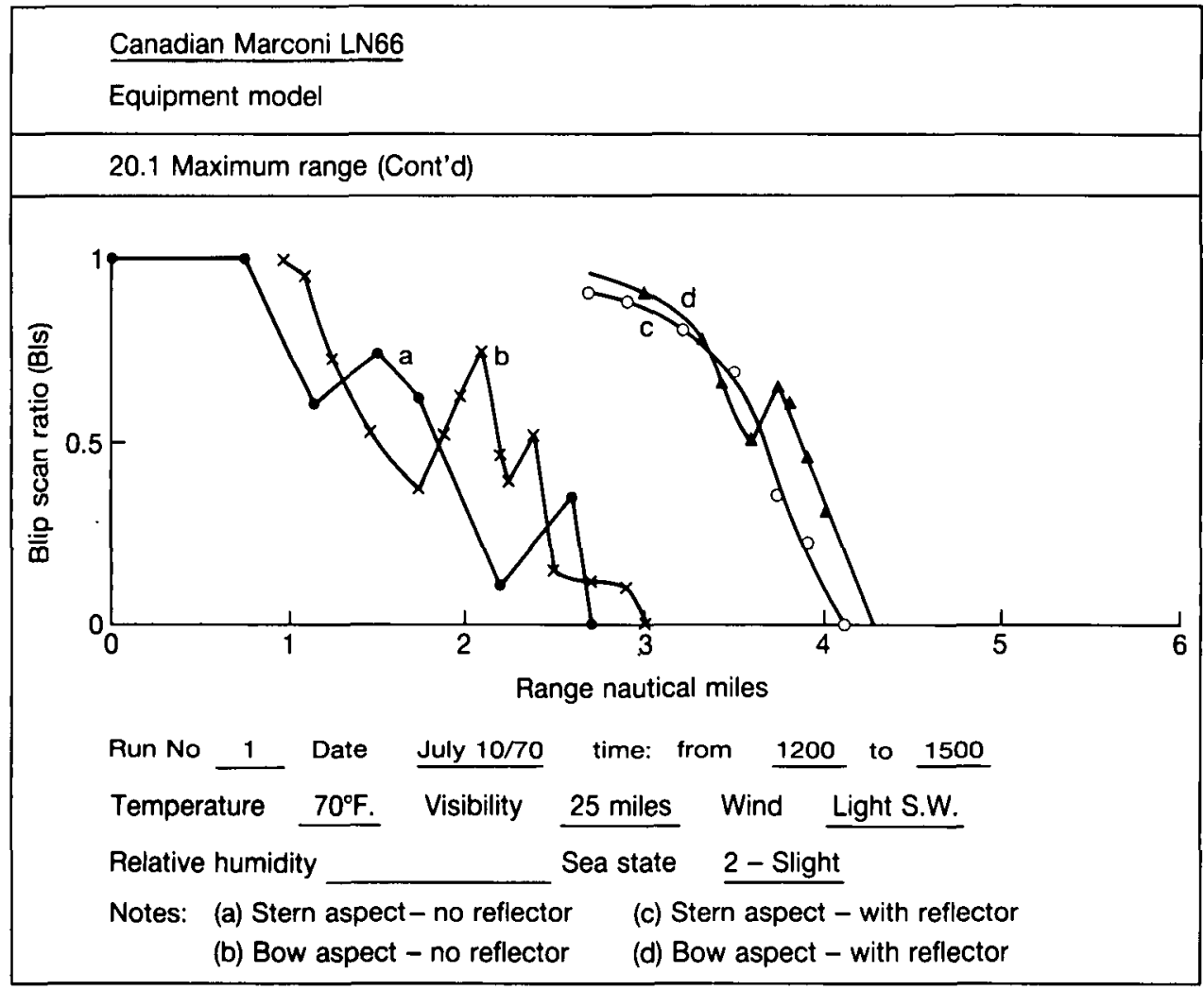

Fig. 1.

Even so, a correction of up to times 15 or $11.8 \mathrm{~dB}$ is referred to on top of the $7-10 \mathrm{~dB}$ given earlier.

The practice of simulating large ships by putting simple, directional corner reflectors onto motor launches or fishing boats is not to be recommended as it will produce very misleading results.

Variations due to ducting also produce major changes in long-range performance where long range is a relative term and may be only to miles for a small horizon range applicable to lower scanner and low target heights (sec run 2 of Squircs' trials on a Canadian LN66 radar in Fig. 3 compared to runs 1 a and $1 \mathrm{c}$ in Fig. I taken 4 days carlicr). The best way to overcome this problem is to carry out trials to assess the absolute radar performance (and if necessary relative performance between a selection of radars, sec Williams, $1980^{7}$ ) well inside the diffraction zone for surface radar and also clear of the fresnel nulls (shown in Fig. 2). Only in this way can true radar performance be assessed without strong environmental corrupting influcnces, even though these may well dominate a real surface radar situation to produce highly variable results.

It is then possible to state that 'Set $A$ ' has $35 \mathrm{~dB}$ spare performance on a one square metre target sphere whilst 'Set B' has $32 \mathrm{~dB}$ spare performance on the same sphere at the same range. If this is carried out then agreement with theoretical predictions may well be within a few $\mathrm{dB}$ providing all system losses are carefully accounted for; otherwise the errors brought about by poor target definition, poor estimation of losses and multipath, may well give rise to positive errors of $15 \mathrm{~dB}$ or more. 
Kelvin Hughes 17/12

Equipment Model

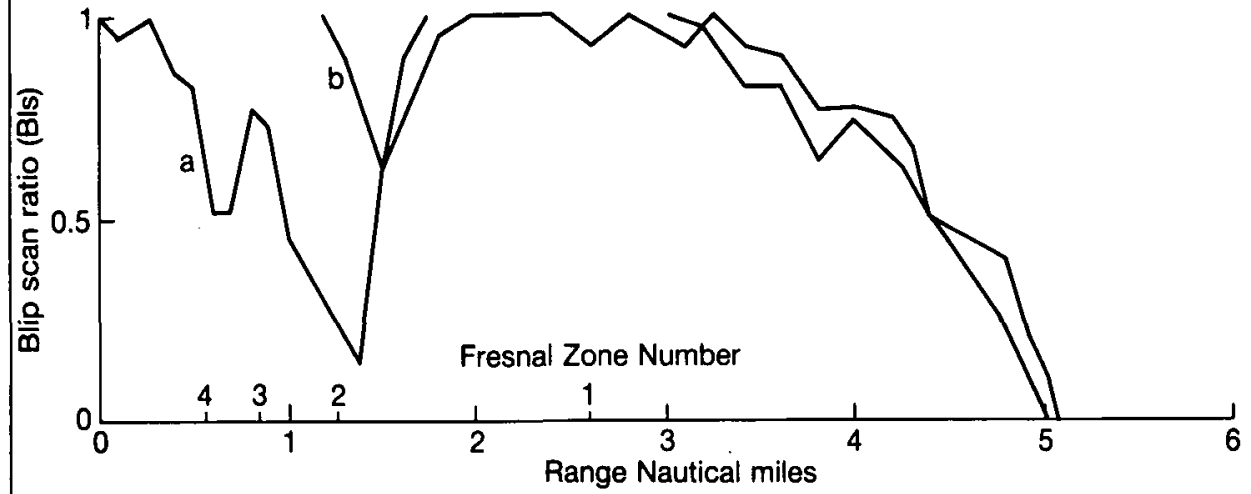

Run No 1 Date July 28/70 time: from $\underline{1300}$ to $\underline{1447}$

Temperature $\quad \underline{74^{\circ} \mathrm{F}}$ Visibility $\underline{5 \text { miles Wind SW } 15-20 \mathrm{mph}}$

Relative humidity Sea state 3 -moderate

Notes: (a) Stern aspect - with reflector (b) Bow aspect - with reflector

Fig. 2.

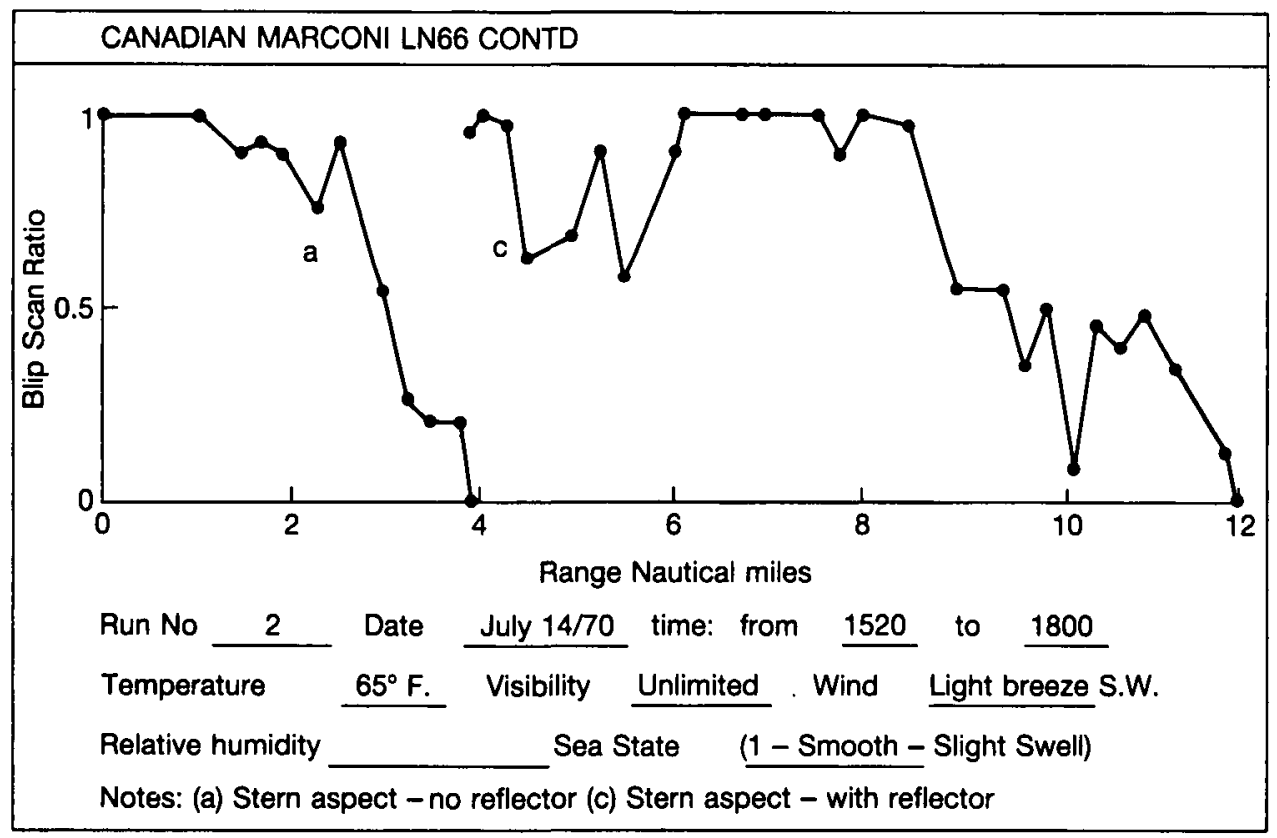

Fig. 3 . 
1 Blake, L. V. (1969) NRL Report 6039.

2 Skolnik, M. I. (1980). Introduction to Radar Systems, 2nd Ed. McGraw Hill, New York.

${ }^{3}$ Kerr, D. E. ed. (1951). Propagation of Short Radio Waves. MIT Radiation Series vol. 13. McGraw Hill, New York.

${ }^{4}$ Squires, D. J., Ward, D. W. and Porter, J. G. (1971). Marine Evaluation Programme 'Radar'. Industrial Development Branch. Department of Fisheries and Forestry Report No. 41 .

5 Williams, P. D. L., Cramp, H. D. and Curtis, K. (1978). Experimental Study of the Radar Cross-Section of Maritime Targets. Proc. IEE Electronic Circuits and Systems, vol, 2. no. 4 (July 1978).

'HMSO (1977). 'Marine Radar Reflector Performance Specification' No. 011-512-01570.

7 Williams, P. D. L. (1980). Medium Term Fading of Radar Targets at Sea with special reference to operation at $3 \mathrm{~cm}$ and $10 \mathrm{~cm}$. Proc. IEE, vol. 127, pt F., no. 3 (June 1980).

\section{'The Navigation of 1985 Trans-Indian Ocean Canoe Voyage' The Author Replies}

With reference to the comments made by John $M$. Luykx, ${ }^{1}$ there is no known direct link between the instruments used on the Trans-Indian Ocean Canoe Voyage and any instruments which may have been used by Viking navigators.

The shadow stick is reputed to have been in use by Greek ship captains at least as early as the first millenium в.C. although it is thought that they went ashore at solar noon to make their observations from a stable base.

The steering board was the simplest device we could construct to make use of the link between the altitude of a celestial body and its direction. No early examples are known. However, neither the board nor its shape nor the peg itself are critical for we often used shadows from the rigging when the sails shaded the area around the helmsman.

In both cases, it is the astronomical principle that is critical. This is likely to have been exploited by ancient navigators through simple devices of many differing designs, some of which may well have been similar to those which we constructed and used.

\section{REFERENCE}

1 Luykx, J. M. (1989). Some notes on the navigation of 1985 trans-Indian ocean canoe voyage. This Journal 42, 309. 